\title{
Targeted gene disruption by use of a group II intron (targetron) vector in Clostridium acetobutylicum
}

\author{
Lijun Shao ${ }^{1,4^{*}}$, Shiyuan $\mathrm{Hu}^{1,4^{*}}$, Yi Yang ${ }^{1}$, Yang $\mathrm{Gu}^{1,2,3}$, Jun Chen ${ }^{1,2,3}$, Yunliu Yang ${ }^{1,2,3}$, Weihong Jiang ${ }^{1,2}$, \\ Sheng Yang ${ }^{1,2,3}$ \\ ${ }^{1}$ Laboratory of Molecular Microbiology, Institute of Plant Physiology and Ecology, ${ }^{2}$ Research Center of Industrial Biotechnology, \\ Shanghai Institutes for Biological Sciences, Chinese Academy of Sciences, Shanghai 200032, China; ${ }^{3}$ Huzhou Research Center of \\ Industrial Biotechnology, Shanghai Institutes for Biological Sciences, Chinese Academy of Sciences, Huzhou 313000, China; ${ }^{4}$ Gradu- \\ ate School of the Chinese Academy of Sciences, Beijing 100080, China
}

Cell Research (2007) 17:963-965. doi: 10.1038/cr.2007.91; published online 6 November 2007

\section{Dear Editor:}

Clostridium acetobutylicum, a gram-positive, anaerobic, spore-forming bacterium, is capable of using a wide variety of carbon sources to produce acetone, butanol and ethanol. To improve solvent productivity of $C$. acetobutylicum, metabolic engineering is considered as a useful tool in developing strains with industrially desirable characteristics. However, to date, there are few useful methods for genetic manipulation of $C$. acetobutylicum, especially for gene disruption. To our knowledge, two types of vectors, including non-replicative and replicative integrative plasmids, have been developed for gene-inactivation in C. acetobutylicum. By using non-replicative integrative plasmids, buk and solR genes of C. acetobutylicum were inactivated $[1,2]$. However, due to their low frequencies of transformation and recombination, the non-replicative integrative plasmids are usually transformed at less than 1 integrative transformant per mg plasmid DNA. To obtain the integrative mutant, it may require higher transformation frequencies up to $10^{5}$, but the typical transformation frequencies were reported at $10^{3}[3]$. Harris et al. described the construction of a replicative integrative plasmid pETSPO and its application in the disruption of gene $s p o 0 A$ which could not be inactivated by using the non-replicative inte-

\footnotetext{
${ }^{*}$ These authors contributed equally to this work. Correspondence: Sheng Yang ${ }^{1}$, Weihong Jiang ${ }^{2}$

${ }^{1}$ Tel: +86-21-54924173; Fax: +86-21-54924015; Email: syang@sibs.ac.cn

${ }^{2}$ Tel: +86-21-54924172; Fax: +86-21-54924015; Email: whjiang@sibs.ac.cn
}

grative plasmid [4]. With the functional replication origin in C. acetobutylicum, pETSPO increases opportunity for homologous recombination, but it is still time-consuming to screen for double crossover integration. Therefore, a more efficient tool for targeted gene inactivation in the $C$. acetobutylicum is much needed.

Recently, a new strategy was developed to construct gene inactivation mutants by using group II intron-based Targetron technology. The mobile group II intron, originating from the Lactococcus lactis L1.LtrB intron, has been successfully used in a wide range of bacteria including Clostridium perfringens [5]. Without a proper replicon and/or promoter, the targetron plasmid pJIR750ai for $C$. perfringens from Sigma Aldrich was not applicable for gene disruption in the $C$. acetobutylicum directly (data not shown). Therefore, a modified targetron plasmid pSY6 was created by cloning the L1.LtrB group II intron fragment into the pIMP1-ptb, which was an E. coli-C. acetobutylicum shuttle vector containing a $p t b$ (phosphotransbutyrylase) promoter [6].

The gene buk, encoding the butyrate kinase, catalyzes the production of butyrate, and the gene solR located on the megaplasmid of the strain, encodes a putative repressor of solvent formation genes [7, 8]. pSY6-buk and pSY6-solR vectors, constructed based on pSY6 (Figure 1A and Supplementary information, Figure S1), were electroporated into C. acetobutylicum ATCC 824, respectively. Then, the cells were incubated overnight to induce the intron invasion (See Supplementary information, Materials and Methods). The overnight cultures were spread onto CGM medium (25 $\mu \mathrm{g} / \mathrm{ml}$ erythromycin) and the transformants were analyzed 


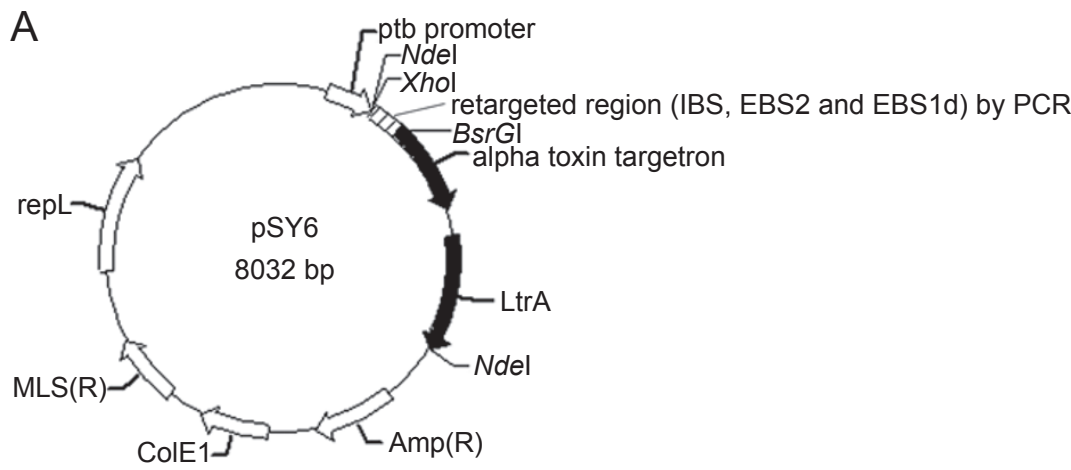

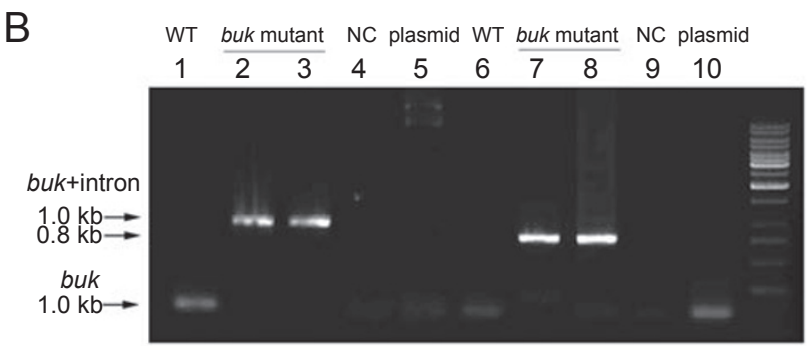

WT solR mutant NC plasmid WT solR mutant NC plasmid

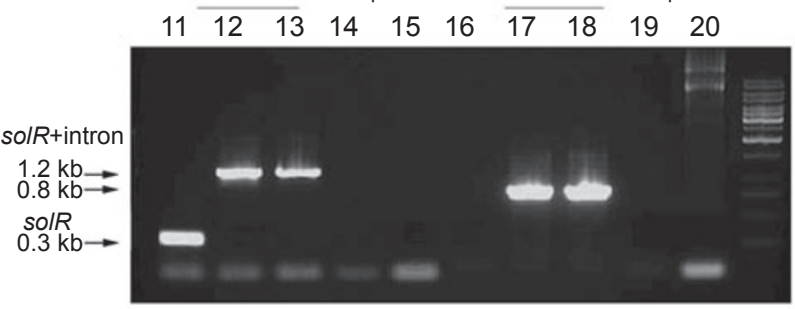

C

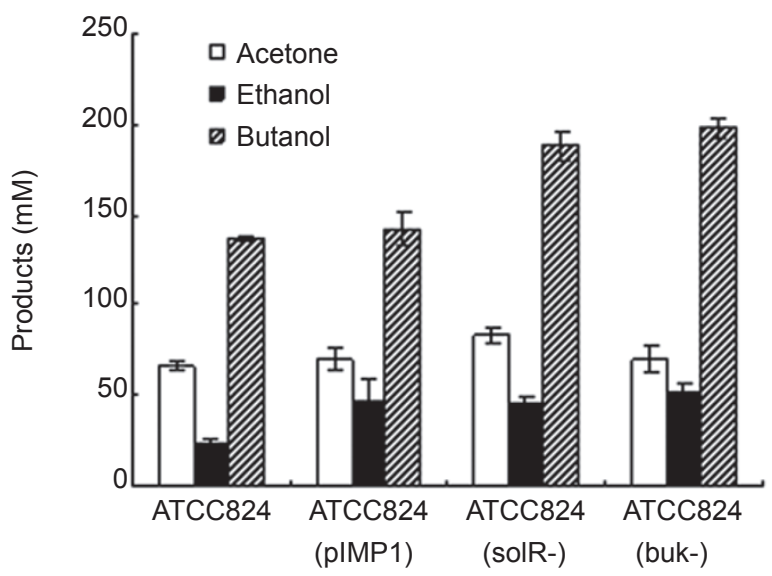

D

\begin{tabular}{|c|c|c|c|c|c|}
\hline Plasmid & DNA $(\mu \mathrm{g})$ & $\begin{array}{l}\text { Number of } \\
\text { transformants }\end{array}$ & $\begin{array}{l}\text { Insertion } \\
\text { frequency }(\%)\end{array}$ & $\begin{array}{l}\text { Number of } \\
\text { insertions/ } \mu \mathrm{g} \text { DNA }\end{array}$ & Reference \\
\hline pJC4BK & 15 & 13 & - & $<1$ & {$[1]$} \\
\hline pDHSR & 4 & 0 & 0 & 0 & This study \\
\hline pSY6-buk & 1 & 770 & $62 \%$ & 474 & This study \\
\hline pSY6-solR & 1 & 450 & $25 \%$ & 113 & This study \\
\hline
\end{tabular}

Figure 1 (A) The targetron plasmid pSY6 for gene disruption in C. acetobutylicum.pSY6 is a derivative tagetron plasmid which was constructed by inserting the alpha toxin targetron and ltrA gene from pJIR750ai into the E. coli-C. acetobutylicum shuttle plasmid pIMP1-ptb and it can be generated into pSY6-buk and pSY6-solR by modifying the retargeted region(IBS, EBS2, and EBS1d). IBS: intron binding site; EBS2: exon binding site 2; EBS1d: exon binding site 1/ ; ColE1: ColE1 origin of replication; MLS(R): macrolide-lincosamide-streptogramin B resistance gene; $\operatorname{Amp}(\mathrm{R})$ : ampicillin resistant; repL: the origin of replication from pIMP1. (B) Confirmation of buk and solR gene disruption in the genome of $C$. acetobutyliucm by PCR. WT: the genome of wild type $C$. acetobuylicum 824; NC: negative control; plasmid: the targetron plasmid pSY6-buk and pSY6-solR for buk and solR disruption, respectively; lanes 1-5, using primers (buk-for-s49/50 and buk-rev-s49/50) flanking the 49/50 site of gene buk; 6-10, using primers (buk-rev-s49/50 and buk49/50s-EBS2) to perform the junction PCR; lanes 11-15, using primers (solR-for-a468/469 and solR-reva468/469) flanking the 468/469 site of gene solR; 16-20, using primers (solR-for-a468/469 and solR-a468/469-EBS2) to perform the junction PCR. Arrows indicate the intron insertion in the mutant. The bands at the bottom of the gel appear as primer dimmers. (C) Comparison of the solvent production between the mutants and wild type strain. (D) Gene insertion efficiencies of non-replicative integrative plasmids DNA and targetron plasmids DNA. 
using colony PCR (See Supplementary information, Figure $\mathrm{S} 2)$. The PCR results showed that the intron insertion frequency was $62 \%$ for buk mutant and $25 \%$ for solR mutant, respectively. However, no transformant was obtained with the non-replicative integrative plasmid (Figure 1D). To further verify the targeted gene inactivation, DNA sequencing of the PCR products amplified from genomic DNA of mutants (Figure 1B) confirmed the presence of L1.LtrB intron integrated in the sense strand at the site between $\mathrm{nt}$ 49 and 50 of buk from its translation start codon and in the antisense strand at the site between nt 468 and 469 of solR from its translation start codon, respectively (See Supplementary information, Figures S3 and S4). Information on all bacterial strains, plasmids and PCR primers could be found in Supplementary information, Table S1.

Solvent production of the mutants was determined by fermentation analysis to identify the phenotype changes caused by the gene inactivation (See Supplementary information, Figure S5). As indicated in Figure 1C, buk mutant and solR mutant produced $44 \%$ and $37 \%$ more butanol than wild type, respectively. These results were consistent with the previously published data $[7,8]$.

Here, we reported the construction of targetron plasmid pSY6 and its successful application in gene-inactivation in $C$. acetobutyliucm, which provides a new genetic tool for gene disruption in this anaerobic organism. The group II intron can insert into defined sites at high efficiencies through a mechanism termed retrohoming independent of homologous recombination [5], which circumvented the low frequency of homologous recombination in C. acetobutylicum. The plasmid pSY6, containing the replicon from pIMP1, could be cured by repeated transferring in the fresh CGM medium without antibiotics [9]. Therefore, it is possible to construct multiple knock-outs consecutively in $C$. acetobutylicum because no selection marker will remain in the plasmid-cured mutant. As a conclusion, this new technology could improve the efficiency of genetic manipulation in C. acetobutylicum and has the potential to be widely applied in the metabolic engineering of $C$. acetobutylicum. During the revision of this manuscript, Heap et al. also adapted a mutagenesis system based on the mobile group II intron from the ltrB gene of Lactococcus lactis (L1.ltrB) to function in clostridial hosts [10]

\section{Acknowledgment}

We thank Prof Peter Duerre (University of Ulm, Germany) for his guidance and support, Prof Eleftherios T Papoutsakis (Northwestern University, USA) for offering pIMP1, pIMP1-ptb and pAN1, Dr Li Chen (Sigma-Aldrich, China) for recommending the targetron system, and Prof Alan M Lambowitz, Dr Jun Yao (University of Texas at
Austin, USA), Drs Yinhua Lu and Yu Jiang (Institute of Plant Physiology and Ecology, Shanghai Institutes for Biological Sciences, CAS, China) for helpful suggestions. This work was funded by National Basic Research Program of China (973: 2007CB707803), High-tech Research and Development Program of China (863: 2006AA02Z237), the Knowledge Innovation Program of the Chinese Academy of Sciences (KSCX2-YW-G-007) and China-Germany collaboration project "Systembiology and Biosystems Engineering". Sheng Yang was funded by Dupont Young Professor Award.

\section{References}

1 Green EM, Boynton ZL, Harris LM, et al. Genetic manipulation of acid formation pathways by gene inactivation in Clostridium acetobutylicum ATCC 824. Microbiology 1996; 142 ( Pt 8):20792086.

2 Nair RV, Green EM, Watson DE, Bennett GN, Papoutsakis ET. Regulation of the sol locus genes for butanol and acetone formation in Clostridium acetobutylicum ATCC 824 by a putative transcriptional repressor. J Bacterial 1999; 181:319-330.

3 Nakotte S, Schaffer S, Bohringer M, Durre P. Electroporation of, plasmid isolation from and plasmid conservation in Clostridium acetobutylicum DSM 792. Appl Microbiol Biotechnol 1998; 50:564-567.

4 Harris LM, Welker NE, Papoutsakis ET. Northern, morphological, and fermentation analysis of spo0A inactivation and overexpression in Clostridium acetobutylicum ATCC 824. J Bacterial 2002; 184:3586-3597.

5 Chen Y, McClane BA, Fisher DJ, Rood JI, Gupta P. Construction of an alpha toxin gene knockout mutant of Clostridium perfringens type A by use of a mobile group II intron. Appl Environ Microbial 2005; 71:7542-7547.

6 Lee SY, Bennett GN, Papoutsakis ET. Construction of Escherichia-coli Clostridium-acetobutylicum shuttle vectors and transformation of Clostridium-acetobutylicum strains. Biotechnol Lett 1992; 14:427-432.

7 Harris LM, Desai RP, Welker NE, Papoutsakis ET. Characterization of recombinant strains of the Clostridium acetobutylicum butyrate kinase inactivation mutant: need for new phenomenological models for solventogenesis and butanol inhibition? Biotechnol Bioeng 2000; 67:1-11.

8 Harris LM, Blank L, Desai RP, Welker NE, Papoutsakis ET. Fermentation characterization and flux analysis of recombinant strains of Clostridium acetobutylicum with an inactivated solR gene. J Ind Microbiol Biotechnol 2001; 27:322-328.

9 Lee SY, Mermelstein LD, Papoutsakis ET. Determination of plasmid copy number and stability in Clostridium acetobutylicum ATCC 824. FEMS Microbiol Lett 1993; 108:319-323.

10 Heap JT, Pennington OJ, Cartman ST, Carter GP, Minton NP. The ClosTron: A universal gene knock-out system for the genus Clostridium. J Microbiol Methods 2007; 70:452-464.

(Supplementary Information is linked to the online version of the paper on the Cell Research website.) 